\title{
A Collaborative Educational Intervention Integrating Biology and Physics in Radiation Oncology: A Design Research Case Study
}

\author{
Monica W. Tracey, Michael Joiner, Sara Kacin, Jay Burmeister \\ Wayne State University, United States
}

\begin{abstract}
Instructional design focuses on solving problems in a multitude of contexts. As such, designers are investigators, gathering evidence to optimally design solutions to learning problems within the identified context. The challenge described in this case study was the need to create an educational activity to promote interaction and collaboration among an interdisciplinary participant group comprised of physicians, radiobiologists, and radiation physicists. Based on the premise that interdisciplinary medical research collaboration requires a shared understanding of authentic problems from multiple perspectives, this design research case documents the design and implementation of an online case study incorporating collaborative inquiry in interdisciplinary teams with the intended outcome of building or strengthening interdisciplinary communication skills. Contextual factors including the design team and design process - influencing the design of the activity are documented. Results indicate that using an interactive online case study as the basis for collaborative inquiry in small, interdisciplinary teams followed by a summative, large group discussion resulted in (1) evidence-based treatment decisions based on the data supplied in the case study and (2) participation of all disciplines in team interactions. Outcomes also indicated the building or strengthening of interdisciplinary communication skills and the understanding of the value and contribution of all three fields to radiation oncology treatment resulted in the participation of the online case study.
\end{abstract}

Keywords: Collaborative educational intervention; Design research; Instructional design; Conjecture mapping; Interdisciplinary teams; Interactive online case study

\section{Introduction}

Instructional design focuses on solving problems in a multitude of contexts. As such, designers are investigators, gathering evidence to optimally design solutions to learning problems within the identified context. The challenged described in this case study was the need to create an educational activity to promote interaction and collaboration among adult course participants. The challenging context was the interdisciplinary make-up of the group. How does a design team create an innovative collaboration-based educational activity for an interdisciplinary group comprised of physicians, radiobiologists, and radiation physicists? This case study documents the iterative design of a collaboration-based educational activity, the Virtual Hospital (VH), and its impact on promoting interaction among physicians, radiobiologists, and radiation physicists while solving problems. We begin with a little background of the content and context. 
Cancer impacts most people one way or another in the United States. Treatment for many of those effected includes radiation therapy. As a result, the number of patients receiving radiation therapy, as a component of their primary cancer treatment, is forecast to increase by more than $20 \%$ over the next decade to almost 600,000 patients per year (Smith, Haffty, Wilson, Smith, Patel, \& Buchholz, 2010). Yet, decreasing emphasis on radiation research over the past decade is making it more difficult to provide oncologists and researchers with the best training. Additionally, there is a lack of integration of this knowledge with medical physics which is now also required in the clinical practice of radiotherapy for cancer (Dynlacht, Zeman, Held, Deye, Vikram \& Joiner, 2015).

In an effort to address this problem, the National Cancer Institute (NCI), a division of the National Institute of Health $(\mathrm{NIH})$ awarded a five-year interdisciplinary grant supporting the design and research of an innovative and advanced 6-day educational intervention for all of the disciplines within oncology. Integrating radiobiology with radiation physics, this intervention demonstrates how to apply state-of-the-art knowledge in the planning and delivery of modern image-guided radiotherapy combined with other modalities. Integrating the content in these two disciplines is not the only issue; there is also a deficiency in collaboration at all levels within these disciplines. Radiobiologists and radiation physicists often do not collaborate on methods of treatment or in research. As a result, it was imperative that the design team incorporate active collaboration-building activities in an effort to integrate the content while teaching collaboration skills to those in each of the disciplines.

The purpose of this case study, part of a five-year, grant-supported research study on the design and implementation of the educational intervention and its components, was to document the iterative design of a collaboration-based educational activity, the Virtual Hospital (VH), and its impact on promoting interaction among physicians, radiobiologists, and radiation physicists while solving problems. Working within a design research framework, the research questions that guided this case include:

1. Did the design of the learning activities in the Virtual Hospital lead to the mediating processes?

2. Did the mediating processes support the learning outcomes?

\section{Case Background}

Advancement in Radiation Oncology depends on the collaborative efforts of the physician, biologist, and physicist. When each of these professionals understand the other two professionals' contributions to the overall care of the patient, three sides of a research and practice triangle are formed which makes each of these researcher's and/or clinician's work more effective and translational. The challenge was to create a collaborative educational environment, bringing these interdisciplinary groups together in an effort to assist each in learning how to speak the same language, appreciate the specialized skills and knowledge of the other disciplines, and engage in activities that foster collaborative investigation.

To be most effective, this educational environment must provide more than cutting-edge information. Such environments already exist within the annual meeting programs of several national/international organizations. This environment is unique in that it draws its educational design from those with expertise in research on instructional design, education and learning. Medical researchers and practitioners should no more expect to successfully create and design 
such an educational environment than educational researchers should expect to successfully conduct medical research. But together we hoped to create an educational environment in which both the content and educational designs were visionary and innovative. This was the inspiration behind the submission of an $\mathrm{NCl}$ R25 funded grant to create an educational intervention entitled Integration of Biology and Physics in Radiation Oncology (IBPRO).

Once funded, the challenge of creating this environment became an immediate reality. The design team had less than a year to design an innovative six-day, face-to-face educational course; appeal to three different specialties in radiation oncology; and prepare to offer cuttingedge information while promoting collaboration. The setting is a conference center in a Midwestern School of Medicine with a large projector and screens in the front of the room, and with eight 'pods' around the circumference of the room that house projectors and screens, and oval tables that can comfortably seat 6-8 individuals in a group. Per NIH requirements, the course would run eight hours each day, plus one hour for lunch and host 40 participants the pilot year, year one, and 50 participants each subsequent year. Stakeholders included the Co$\mathrm{PI}^{\prime} \mathrm{s}$, a biologist and instructional designer, Co-Investigators, a physicist and instructional designer with expertise in assessment and evaluation, representatives of $\mathrm{NCl}$, the funding agency, additional instructional designers, graphic designers, desktop publishers, other consultants, and the participants - a mix of physicists, biologists, and physicians in radiation oncology. Since the funding of this grant, the course has been offered 3 times to a total of 140 participants. The remainder of this article focuses on the design and implementation of one instructional activity implemented during the six-day course. Initially designed as a collaborative icebreaker activity, and now through three redesign iterations, the Virtual Hospital has evolved into a critical component of the course.

\section{Literature Review}

As Educational Technology is concerned in part with is the study of facilitating learning by creating and using appropriate processes (Januszewski \& Molenda, 2008), this case study reports on the application of two theories and a method of design used to create an educational approach to learning. By utilizing the Layers of Negotiation Model in the design of this educational intervention while applying the theories of collaborative learning and active learning to the educational activity, we studied the practice of designing and implementing an approach to learning.

Collaborative Learning. Collaborative learning is defined as an opportunity where learners "develop, compare, and understand multiple perspectives on an issue" (Karagiori \& Symeou, 2005 , p.21). The origins of collaborative learning, a popular concept in education, first emerged in the 1950s and 1960s by a group of British secondary school teachers and a Biologist studying medical education (Brufee, 1984). Focusing on traditional patient diagnosis methods, medical students clustered around a patient with a doctor, the biologist researcher requested the medical students to examine the patient together, discuss the case and come to consensus on a single diagnosis that they could all agree. Results indicated that students learning diagnosis collaboratively obtained good medical judgment faster than those working alone. The culmination of this ten-year study on collaborative learning in medical education demonstrated that important foundations in successful medical practice are better learned in small groups (Abercrombie, 1960). One benefit of learning collaboratively is the ability to transfer these skills to other contexts. 
Radiation Science acknowledges the need for collaborative learning in an effort to advance radiation research and improve cancer patient care. Sullivan (2000) calls for Team Science, a multidisciplinary approach to break down traditional barriers, foster collaborative interactions and harness new technologies for hypothesis-driven research to advance the field of radiation oncology. He further stated that in order to bridge the gaps in research and practice in the field, the NIH must facilitate and support multidisciplinary efforts. As such, a multidisciplinary approach to cancer management involving surgical, radiation, and medical oncologists working in collaboration with other health care professionals has become the recognized standard of care (Hirsch, Bishop, Dad, Signh, Slanetz, 2009). In 2002 the National Institutes of Health (NIH) identified interdisciplinary collaboration as a major priority of all future research efforts (Zerhouni, 2003) in turn funding this research as it focused on interdisciplinary instruction delivered in a collaborative activity. However, Dynlacht et al. (2015) indicate that even when collaborations do occur between biologists and physicists, there is a lack of common language and common training which may inhibit research progress. They maintain that biologists need to know about radiation oncology in general and that both biologists and physicists should be familiar with the types of cancer that causes the most deaths due to poor tumor control. IBPRO is designed to educate all three disciplines through keynote lectures given by experts in biology, physics, and clinical oncology, while educating participants on how to collaborate while providing collaborative learning activities.

Active Learning. Principles of the constructivist learning strategy, active learning, were employed in the design and development of the overall educational intervention including the $\mathrm{VH}$. Within the theoretical framework of constructivism, active learning is built on the premise that "learning is an active process occurring in realistic and relevant situations" (Richey, Klein \& Tracey, 2011). Active learning is an application of constructivist theory, which maintains that learners construct their knowledge based on their personal interpretation of experience; they explore ideas from multiple perspectives and are active agents in the learning process. Active learning goes beyond processing information provided by materials or instructors. As such, it involves more than physically participating in predetermined learning activities. It includes interacting with information at a high level, elaborating upon this information, and interpreting it in light of one's previous knowledge and experiences (Perkins, 1992). The participants in this educational six-day intervention bring extensive knowledge and experience to the course. The team determined the design solution must incorporate collaborative active learning activities to encourage the sharing of knowledge and experience with others while promoting collaboration.

Layers of negotiation model. One premise of this study was to examine constructivist ID principles for designing interdisciplinary instruction as well as applying constructivist learning processes for the collaborative activities. As such, the constructivist ID principles of the Layers of Negotiation Model were the foundations framing design activities. The ID team determined that in an effort to ensure success while designing within this complex context and meeting the funding agency and end user's expectations, they needed to employ the constructivist ID Layers of Negotiation Model (Cennamo, 2003). This model employs stakeholders in the project and focuses on the process of knowledge construction using reflection, the examination of information multiple times for multiple purposes, and the social negotiation of shared meanings (Cennamo, 2003). The immersion and collaboration with the design team and the subject matter expert (SME) stakeholders created trust and the opportunity for iterative feedback during the design of the instructional materials and strategies. One output of implementing this model was weekly update design team meetings (Tracey, 2015). These gatherings provided a venue for all stakeholders to make instant decisions and revisions of process and instructional materials. To continue ongoing collaboration efforts, Google docs were used to record content, 
design ideas and for revisions in-between weekly meetings. Incorporating the Layers of Negotiation Design Model permitted iterative cycles of design and observation while creating instructional materials (Statwicz \& Stevens, 2008).

\section{Methodology}

The funding from $\mathrm{NCl}$ along with the singular and naturalistic nature of the project provided a setting conducive for employing a design research case study. This section will first describe the important contextual factors including the design research approach, important theoretical foundations of the project, the design team, the course participants, the design process, and the initial design solution. Finally, it describes the data collection and analysis process and resulting design changes based on results from the data.

\section{Design Research}

As design research is a method where the creation of innovative learning environments, including knowledge about how such environments work contextually, are documented and studied, with the goal of enhancing research on knowledge about learning or teaching (Sandoval, 2014), it was advantageous to apply this approach to research the design process and products generated in this study. This case also tested the application of theoretical constructivist instructional design (ID) principles by applying the Layers of Negotiation Model for the design of an active educational intervention, which provided the opportunity for learners to derive meaning from the contextual experience thus constructing their personal knowledge.

\section{Research Approach}

The focus of this research study was on the implementation of a learning environment and how this environment impacted the desired learning outcomes in this setting. Conjecture mapping (Sandoval, 2014) provides a way to articulate the design and theoretical ideas represented in a learning environment and how they are predicted to work together to produce desired outcomes. By applying design and learning theories and analyzing the learning outcomes of the participants in this environment we attempted to capture how the identified theories worked together to produce the desired learning outcomes. Conjecture mapping provided a means for us to externalize the connections among learning theory, instructional design, and learning outcomes for the $\mathrm{VH}$.

\section{The Design Team}

Assembling the appropriate design team was critical to the success of this project. Nelson and Stolterman (2012) state that "an appreciative judgment, appreciating what is important to consider and what is not; whose interests need to be taken into account and whose do not; and what level of complexity must be maintained as a substitute for never-ending comprehensiveness... It is within this context, and against this environment, that the design process unfolds," (p.115). The two grant Co-PI's - one a researcher in ID, and the other a researcher in Biology - were key leaders on the team, each bringing their expertise in choosing 
the remainder of the team (Tracey, 2015). Two additional Co-Investigators on the grant were also critical members the design team: a researcher in ID with specific expertise in assessment and evaluation and a researcher in Medical Physics (Figure 1). These four members brought biology and physics content expertise in addition to the needed instructional design expertise (Tracey, 2015).

Additional instructional designers hired to work on the team were colleagues and/or were current graduate students in the Instructional Technology program at the host university. Knowing the depth of the content and the overall complexity of the design project, designers were hired who had a minimum of six years of experience specifically with ill-structured, multilevel projects. One tenet of the layers of negotiation model is continuous reflection on decisions, which requires team members to maintain flexibility, be reflective, while being self-confident in their design judgment (Tracey, 2015). Designers were chosen who had the ability to create ideas, and then have their ideas analyzed, changed and built upon which played a significant role in the success of the Layers of Negotiation design process and in the end product, the activity learning activity.

The biologist and the physicist served as the content subject matter experts (SMEs) while providing access to additional biology and physics researchers, physicians and clinicians to support our design efforts along with a window into the end learners. As the design team proposed ideas, these SME's provided instant critical feedback on the viability of the design. As Figure I illustrates, the project team was comprised of additional members including graphic designers, desktop publishers, computer programmers and website developers who were brought in on an as-needed basis and worked with the designers to develop products (Tracey, 2015).

\section{Course Participants}

A goal of IBPRO is to expose course participants to internationally recognized experts within the fields of cancer biology, radiobiology and radiation physics, who also have track records of effective research and teaching in these fields. IBPRO accepts participants who are researchers and clinicians from any discipline wishing to undertake research in radiation oncology, new researchers wishing to gain knowledge to effectively enter the field, and existing professionals and researchers wishing to refresh and advance their knowledge to state-of-the-art. As such, participants each year overall exhibit similar characteristics, with minor demographic variations. These variations had little impact on the design of the Virtual Hospital.

Year 1, the pilot year had 40 participants with Years 2 and 3 each having 50 course participants. The average age for all of the years was in the mid-40s with males dominating female attendees. Physicists outnumber biologists with and clinicians by a 2:1:1 ratio. Participants held PhDs or PhDs and MDs with extensive professional experience. The experience of the participants required the design team to plan for innovative and intellectually challenging active learning activities. 


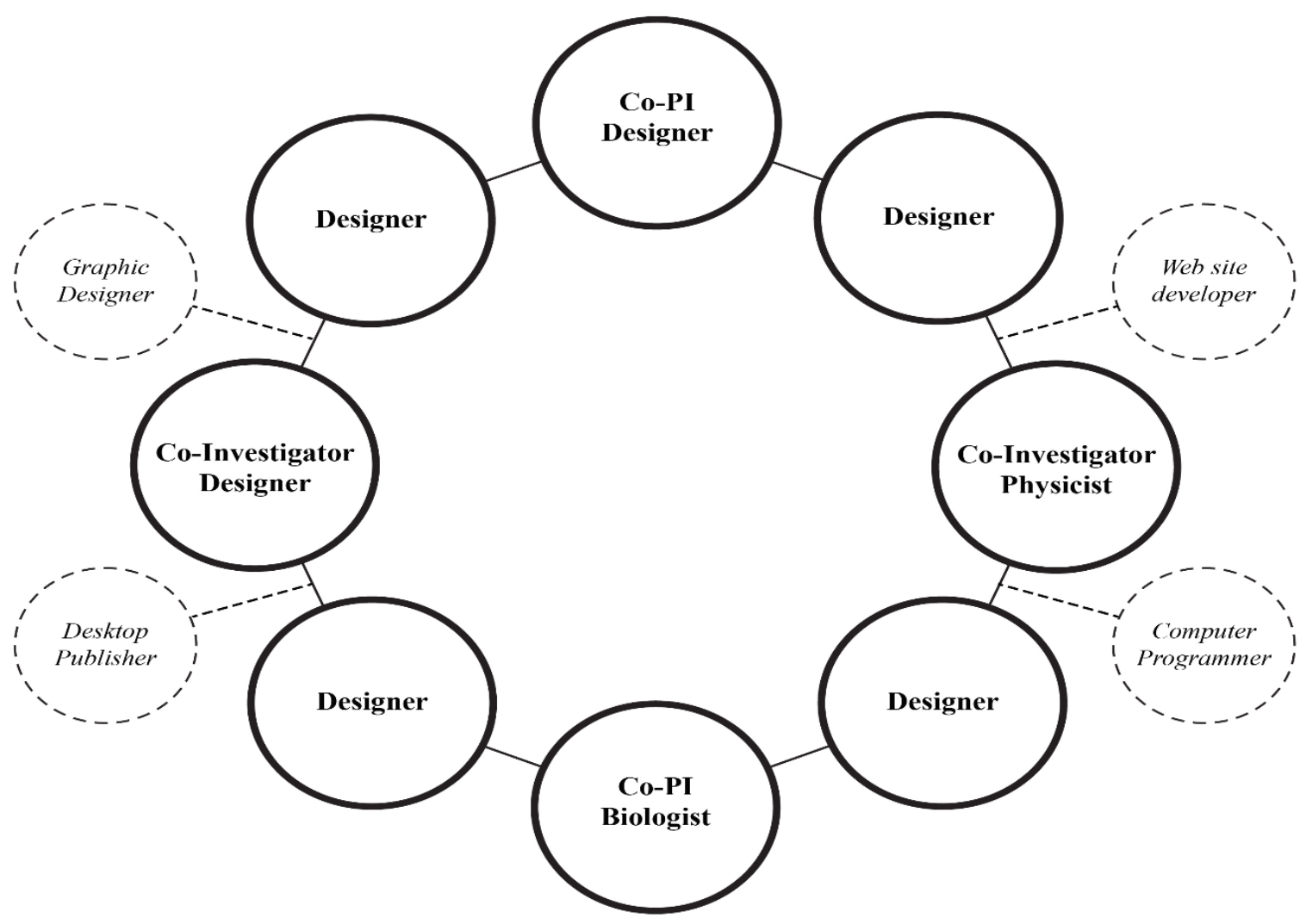

Figure 1. The Design Team

\section{The Design Process}

Design is a process of meaning making (Nelson \& Stolterman, 2012) where things make sense when they are connected and interrelated or presented through relationships in context. Design is rooted in a context framed in time and place and designers must understand the context of the design situation as quickly and thoroughly as possible. The context of this design project included the lack of cross-pollination in biologists, physicists and physicians in radiation oncology who are planning and conducting relevant productive practice and research in this area. Embracing a holistic perspective, simultaneously looking at all of the components of the design problem, postulated the impetus for the team, how it was assembled, how often we met and the method in which we worked. Employing Layers of Negotiation principles for shared meaning; we reflected on what was accomplished and iteratively established new weekly goals. Design constraints including design time restrictions, the imminent final delivery date, a complexity of the overall task and final product, dense content and numerous stakeholders concurrently impeded and accelerated the design.

Collaboration while designing is not a new concept although instructional design activities appear to be mostly completed individually. Some question whether this is the best way to design (Brown, 2009) and if a "radical form of collaboration where designers migrate toward ever-deeper collaboration not just among members of a design team but between the team and the audience it is trying to reach" is needed (p. 58). Brown states that we must look at design as an opportunity to work with the stakeholders and end users, not for them. Business and industry, he contends should think about how more time could be spent doing collaborative, 
procreative work in face-to-face time. Brown argues that this will produce a solid outcome. He believes face-to-face time nurtures relationships and inspires teams and is one of the most precious resources an organization possesses. In terms of design, Brown states that time collaborating in teams should be as productive and creative as possible where each person builds on the ideas of others. This he believes makes design easier, when design is happening in real time and among people who know and trust one another (Brown, 2009).

Design collaboration, however, is not always an easy or intuitive activity. Studying designing in teams, Brereton, Cannon, Mabogunje, \& Leifer (1996) noted that each individual designer requires behaviors of collaboration that include expressing ideas, listening, and negotiating. A design team's process is quite complex and controlled although it may be free flowing (Cross, 2011). Brereton et. al (1996) discovered that designers working in collaborative teams are constantly engaged in numerous activities at different levels and continuously look at alternatives while reflecting, monitoring and modifying their process and course of action. Solutions are created based on the requirements and a constant review of various solution alternatives.

To support collaboration, the design team participated in mandatory 2-hour meetings in one room, around a circular table where an initial idea was introduced to begin the creative design process. Applying the Layers of Negotiation Principles during these meetings strengthened the collaborative process. There were no breaks, cell phones were turned off, and the singular goal of designing by identifying constraints, creating ideas, and building on them was achieved. Each team member was selected due to his or her ability and desire to create an innovative design while having a stake in achieving the final product.

\section{The Virtual Hospital Conjecture Map}

Mapping the conjectures guided the design team in identifying and documenting the theoretical features of the learning environment and mapping out how we predicted these features would work together to produce our desired outcome. The design team began with the initial hypothesis; that interdisciplinary medical research collaboration requires a shared understanding of authentic problems from multiple perspectives. We then illustrated the conjectures that would drive the design team's decisions. These were based on reasoned beliefs, or conjectures that certain features of the designed learning experience would lead participants to engage in particular activities - or mediating processes - that would in turn lead to particular learning outcomes. We believed that using an interactive online case study as the basis for collaborative inquiry in small, interdisciplinary teams followed by a summative, large group discussion would result in the following mediating outcomes: (1) evidence-based treatment decisions based on the data supplied in the case study and (2) participation of all disciplines in team interactions. We then identified the learning outcomes that we believed participants would reach as a result of those mediators. These included building or strengthening interdisciplinary communication skills and understanding the value and contribution of all three fields to radiation oncology treatment (See Figure 2). 


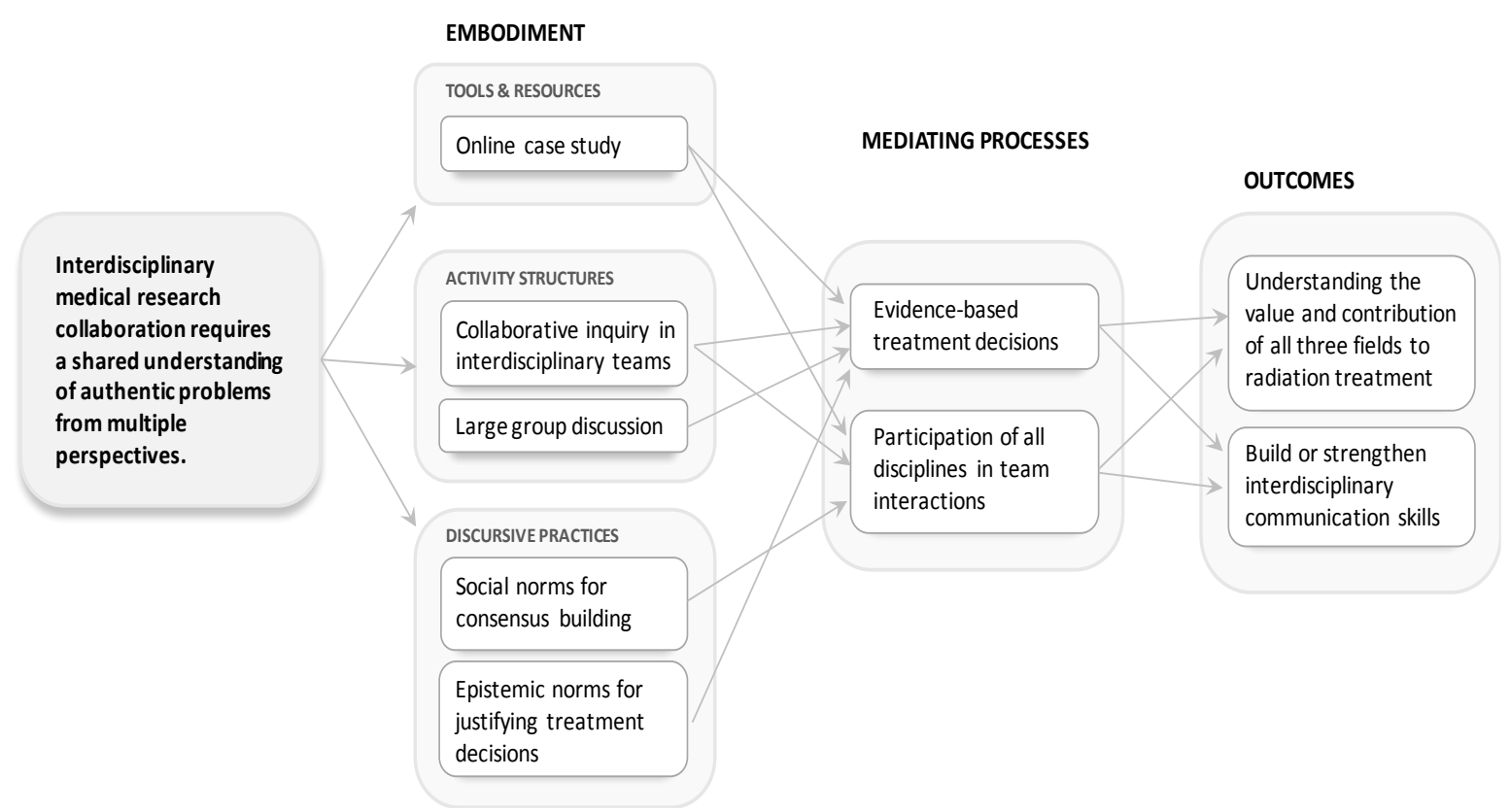

Figure 2. The Conjecture Map

\section{The Design Solution}

The Virtual Hospital activity ushered the beginning of each eight-hour course day followed by a Structured Debate, and two keynote presentations, one focusing on Physics, and one on Biology. The afternoon opened with a Networking Activity followed by a third keynote presentation from a clinician focusing on clinical applications of the day's theme. The remainder of the afternoon included various structured activities along with a Protocol Development Activity where groups work throughout the entire week to design a clinical research study, implementing one or more radiation oncology interventions to evaluate the effects on health outcomes. The remainder of this article focuses on the design and implementation of the Virtual Hospital.

The initial design. Year 1 of the six-day intervention consisted of twelve different cutting-edge themes, two per day, in radiation oncology. For example, Day 1 themes were Cell Death and Long-Term Consequences of Treatment, and Particle Therapy: Protons and High-LET. Biologist and physicist keynote speakers addressed each theme through a presentation, for a total of four keynote presentations per day. (Note: This structure was redesigned in year two and the subsequent years as there are currently three keynote presentations per day.) The design of the Virtual Hospital was in response to the need to promote collaboration while introducing the daily theme thus giving participants the opportunity to collaborate on an interactive case relating to that day's content themes. The Virtual Hospital includes the presentation of a patient case, support documents including scans, images and test results provided in the different Virtual Hospital rooms, and structured questions for the groups to answer.

The case content, including all supporting materials, were placed in an accessible Word document and divided into identified sections to insert into the Virtual Hospital online format. These documents were then delivered to the graphic designer, hired to create the Virtual 
Hospital visual structure and a computer programmer, hired to create the code in order to achieve the end product. Completed products were sent to the web designer who uploaded the files to the Blackboard LMS used for the entire course. It is important to note that the Virtual Hospital activity itself was conducted in the classroom with participants working face-to-face to collaboratively solve the patient case. This activity therefore does not meet the criteria of a virtual reality activity.

To better illustrate the activity, the Virtual Hospital on the first day presented Avery, an eightyear-old pre-pubescent Caucasian male who plays contact sports (pee-wee football). He begins complaining of a headache that gets worse over 1 week, accompanied by nausea/vomiting, and "clumsiness" (occasionally stumbling and losing balance). His mom takes him to the Emergency Room, suspecting that he may have hit his head at football practice and may have a concussion. Numerous tests are administered with the final diagnosis of medulloblastoma determined. Avery is then referred to a pediatric oncologist and radiation oncologist. This case is presented on this day as it involves proton therapy, one of the key themes.

To solve this and subsequent Virtual Hospital cases, IBPRO course participants are evenly distributed into working groups of 6-7 to read and analyze a case while incorporating expertise from all three disciplines (clinical, biology, and physics). The activity begins with participants clicking on a link in the IBPRO course website to access the Virtual Hospital. A new tab opens and participants click on the "Welcome" pin to begin moving through the interactive case (See Figure 3 and 4). In year one, each group member, given a hospital name from popular TV shows (e.g. Chicago Hope, ER, Seattle Grace etc.) was provided an iPad to individually access the Virtual Hospital.

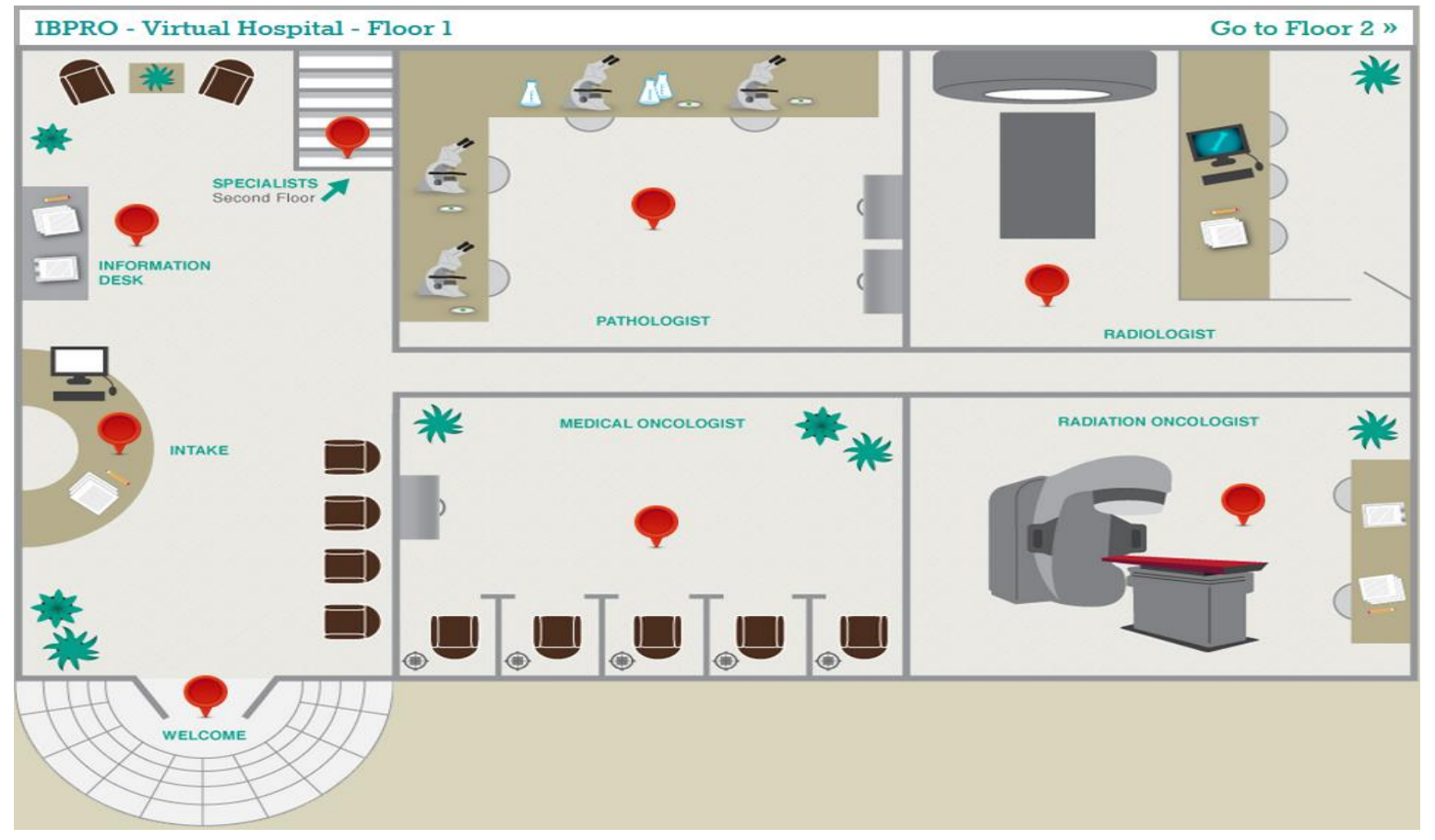

Figure 3. Screenshot of the Virtual Hospital, $1^{\text {st }}$ Floor 


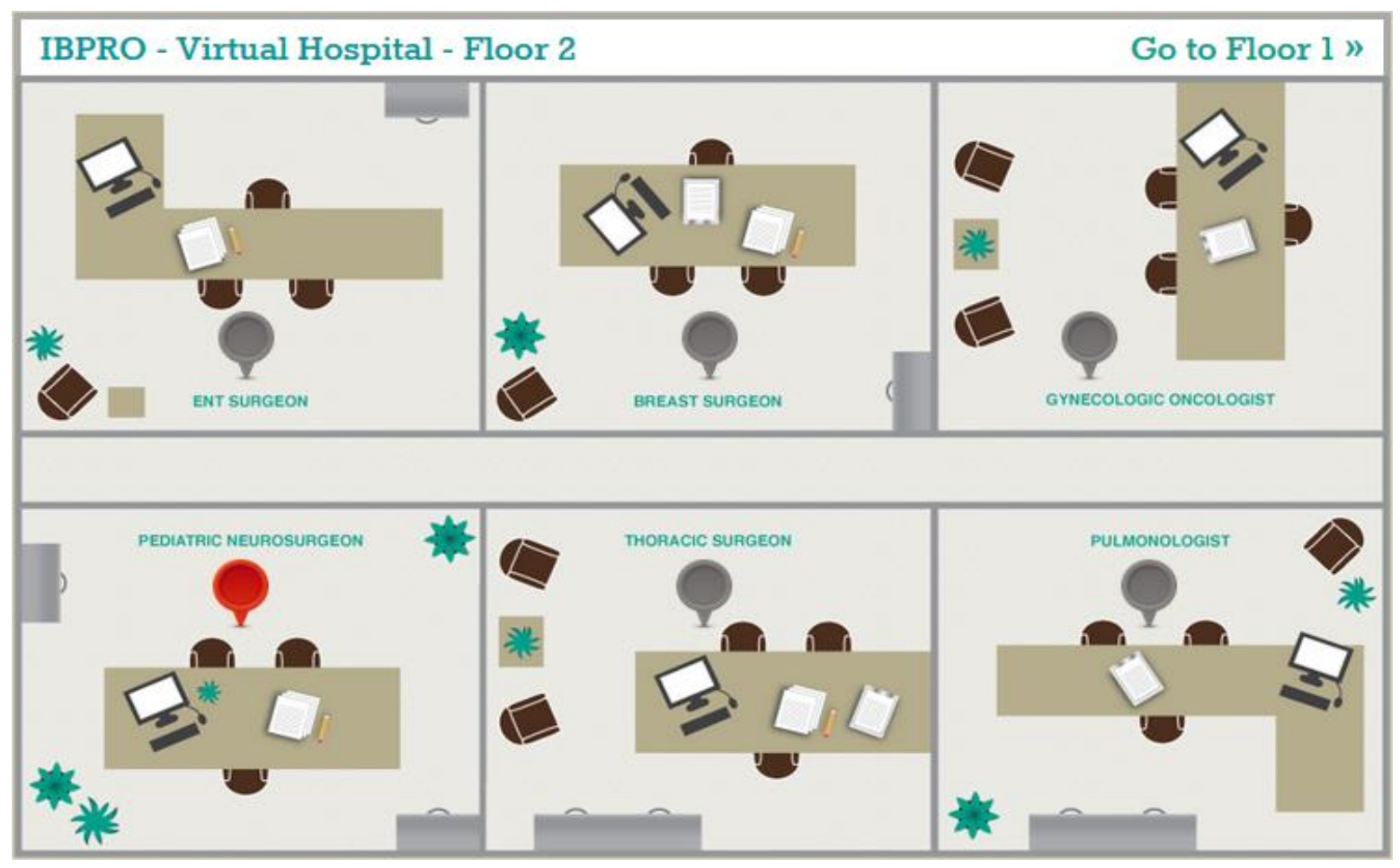

Figure 4. Screenshot of the Virtual Hospital, $2^{\text {nd }}$ Floor

The participants listen to a taped welcome message from a nationally recognized radiation oncologist then are prompted to the intake desk where a brief patient description is presented. Participant groups must decide collectively where to navigate next as they may then choose one or all of six various pins to click on to gather additional information including pathology and medical oncology reports, images from the radiologist, and a discussion of treatment options from the radiation oncologist. The participant groups may also visit the second floor of the Virtual Hospital where a host of specialists present additional patient information. Once participant groups visit their chosen areas of the $\mathrm{VH}$, they respond to the questions regarding the case presented in a separate link (Table 1). Although given paper copies of the questions, upon completion of the case, one member of the group is responsible for submitting the final answers electronically for the entire group on the Blackboard site. After 20 minutes, the participants debrief the questions in one large group with a clinician and subject matter expert for the day's theme.

Table 1. Virtual Hospital (VH) Questions

\begin{tabular}{|c|c|}
\hline Year 1 and 2 Questions & Year 3 Questions (Day 1) \\
\hline Is this cancer "curable"? & $\begin{array}{l}\text { How do you counsel the patient and their } \\
\text { family regarding late effects of radiation } \\
\text { therapy? }\end{array}$ \\
\hline $\begin{array}{l}\text { What is the standard treatment of } \\
\text { practice? }\end{array}$ & $\begin{array}{l}\text { Does that discussion change if you are } \\
\text { treating with protons? }\end{array}$ \\
\hline
\end{tabular}


What is the potential toxicity (side effects)? If treating with photons, what technique would you use?

What are the treatment options?

What is the treatment duration (how long does this treatment take)?

\section{Data Collection and Analysis Processes}

As described earlier, conjecture mapping (Sandoval, 2014) provides a way to externalize the connections among learning theory, learning design, and learning outcomes for the Virtual Hospital. It also supplies an organizing framework for gathering data relevant for evaluating both the design conjectures and theory conjectures that shaped the activity initially and across iterations. This section will discuss processes used to collect and analyze data about the learning experiences that emerged for participants in relation to both sets of conjectures.

First, data was collected via formal and informal channels. Informal data included observations of participants as they engaged in the activity by the design team and subject matter experts. For example, the Virtual Hospital included a large group discussion at the end, which was led by a subject matter expert who provided feedback to each Virtual Hospital team based on their responses to specific case questions. Because the design team was present for this activity on all days it was offered, they were able to observe first-hand whether the teams were making sound, evidence-based treatment decisions based on an expert's appraisal. They shared their observations of the activity with each other daily during the course as well as at a formal debriefing after the course. These observations were noted, synthesized, and incorporated in the analysis of the activity conducted for all design iterations.

As an $\mathrm{R} 25 \mathrm{NIH} / \mathrm{NCl}$ research grant that also offers Continuing Medical Education (CME) credits to participants, our formal data collection activities were designed to ensure compliance with the expectations of our funders and accreditors. In line with these expectations, we asked participants to evaluate every learning activity and keynote speaker via online surveys. These surveys included scale-based ratings and short, open-ended questions that assessed participants' perceptions of their experiences throughout the course. Numerous steps were taken to determine reliability and validity. The first three quantitative questions are required for $\mathrm{CME}$ accreditation, ensuring reliability and validity. The qualitative questions were written by experts in learning and evaluation and then confirmed by subject matter experts. Based on participant feedback in the initial year, we scaled back the number of times we surveyed participants strengthening validity. Additionally, participants had the opportunity to provide open-ended suggestions and none commented that the survey questions themselves as being inaccurate or invalid. Although there were minor procedural changes in data collection from year to year, the core aspects of the Virtual Hospital that were being assessed by participants remained consistent across all three years. These included overall success of the activity in meeting objectives; effectiveness of the activity; educational quality; participants' overall perception of the activity; what they liked the most about the activity; and what suggestions they had to improve it. The one alteration to these topics concerned an item asking participants to describe their perception of the Virtual Hospital. Year 1 participants were specifically asked 
to identify five descriptors of how their small group functioned during the activity, while in Years 2 and 3 they were asked to describe the activity in general. Because improved collaboration is the focus of this learning experience, the design team wanted to understand specifically how participants viewed their team interactions during Year 1 . Having established a baseline sense of collaborative processes during the first iteration, the question was phrased more generally in Years 2 and 3 by asking participants how they would describe the activity in general to another person. By making this change, we could better gauge the importance that participants placed on elements of collaboration in relation to the activity as a whole without using a directive question leading them to focus on this aspect.

Table 2 summarizes data collection procedures specific to the Virtual Hospital activity by collection method and the aspects of mediating processes and learning outcomes that were being assessed. More details about the collection processes, including the wording of specific questions, are included in the next sections as relevant.

Table 2. Virtual Hospital (VH) Data Collection Overview

\begin{tabular}{ccc}
\hline Source of data & Mediating processes & Learning outcomes \\
\hline $\begin{array}{c}\text { Design team and subject } \\
\text { expert observation of the } \\
\text { activity while it was } \\
\text { occurring }\end{array}$ & $\begin{array}{c}\text { Evidence-based treatment } \\
\text { decisions using case study data } \\
\text { Participation and integration of } \\
\text { all disciplines in team } \\
\text { collaboration }\end{array}$ & $\begin{array}{c}\text { Building or strengthening } \\
\text { interdisciplinary } \\
\text { communication skills }\end{array}$ \\
$\begin{array}{c}\text { Responses to open-ended } \\
\text { questions from } \\
\text { participant surveys } \\
\text { addressing the Virtual } \\
\text { Hospital }\end{array}$ & $\begin{array}{c}\text { Participation and integration of } \\
\text { all disciplines in team } \\
\text { collaboration }\end{array}$ & $\begin{array}{c}\text { Understanding the value and } \\
\text { contribution of all three fields } \\
\text { to radiation treatment }\end{array}$ \\
\hline
\end{tabular}

\section{Data Analysis}

As mentioned, the two primary sources of data were qualitative data gathered via participant surveys along with observations of the activity by the design team and SMEs. The analysis process for qualitative data was established in the first year and refined in subsequent years as new data were added to the overall pool of responses. One member of the design team conducted the analysis; the entire team subsequently reviewed the raw data as well as the qualitative results and discussed both at a team meeting. The same team member conducted qualitative analysis for Years 2 and 3 as well, with other team members reviewing raw data and results for these iterations of the course. Thematic analysis (Lichtman, 2006) was the general approach used, with key themes and issues emerging from multiple readings of participants' responses. In many cases, a single response might be coded for more than one category; for example, a response providing suggestions for the Virtual Hospital may have mentioned allowing more time and providing more background information. Thus, it would be appropriate to code it twice as it contained two separate and meaningful pieces of information. Once all data had been categorized for each question, raw counts were tallied and percentages were 
calculated for each category within a given question; these simple statistics were not intended to convert qualitative data into quantitative data, but rather to provide an efficient way to identify trends and patterns in responses that may have gone unnoticed otherwise.

Because these observations were done informally and generally reflected a consensus, they were not formally analyzed but rather summarized by the design team leader for inclusion in the team's assessment of each iteration of the activity.

\section{Research Results and Their Role in the Design Context}

This section will (1) describe our research results in relation to mediating processes and learning outcomes associated with the activity and (2) discuss how these results were used by the design team to make design decisions across iterations of the activity. While it is typical for research papers to present results independent from any discussion of them, we believe that logic of design research requires that they be connected to the design context in order to fully appreciate their meaning and value. A subsequent section of the paper will discuss the design principles that emerged from these contextualized results.

Before we look closely at data in relation to the conjectures that drove the activity's design, it may be helpful to consider how participants rated the $\mathrm{VH}$ on general learning criteria as required for CME accreditation: educational objectives met, activity effectiveness, and educational quality (Table 3). Participants were asked to rate the activity using the following numeric scale: (1) Poor; (2) Somewhat poor; (3) Neutral; (4) Somewhat good; and (5) Excellent. As detailed in, Year 1 had the highest overall averages of the three years (objectives met, 4.75; activity effectiveness, 4.70; and educational quality, 4.67), while Year 3 had the lowest (objectives met, 4.35; activity effectiveness, 4.31; educational quality, 4.35). Scores tended to be consistent across the three criteria in a given year, and the cumulative means for each dimension were also tightly clustered (objectives met, 4.58; activity effectiveness, 4.56; and educational quality, 4.57). Qualitative data also suggested that participants valued the activity; for example, participants were asked to describe the experience of participating in the Virtual Hospital and only four percent of the resulting descriptors from all three years were negative in nature. When participants were asked to provide suggestions to improve the activity, $24 \%$ of all responses were a general positive comment about the activity with no critique (i.e., "keep it up," "great activity, should be continued," etc.).

Table 3. Mean Participant Ratings of the Virtual Hospital (VH)

\begin{tabular}{cccc}
\hline Rating interval & $\begin{array}{c}\text { Objectives met } \\
\text { mean }\end{array}$ & $\begin{array}{c}\text { Activity effectiveness } \\
\text { mean }\end{array}$ & $\begin{array}{c}\text { Educational quality } \\
\text { mean }\end{array}$ \\
\hline Year 1 overall & 4.75 & 4.70 & 4.67 \\
\hline Year 2 overall & 4.54 & 4.53 & 4.55 \\
\hline Year 3 overall & 4.35 & 4.31 & 4.35 \\
\hline Cumulative & $\mathbf{4 . 5 8}$ & $\mathbf{4 . 5 6}$ & $\mathbf{4 . 5 7}$ \\
\hline
\end{tabular}




\section{Mediation Processes: Results and Discussion}

Having established that participants saw the $\mathrm{VH}$ as a valuable educational activity in general, we can now turn our attention to examining the conjectures that drove the design team's decisions. As Sandoval (2014) outlines, design decisions are based on reasoned beliefs, or conjectures that certain features of the designed learning experience will lead participants to engage in particular activities - or mediating processes - that will in turn lead to particular learning outcomes. Returning to Figure 2, the design team believed that using an interactive online case study as the basis for collaborative inquiry in small, interdisciplinary teams followed by a summative, large group discussion would result in the following mediating outcomes: (1) evidence-based treatment decisions based on the data supplied in the case study and (2) participation of all disciplines in team interactions.

Our assessment of the first mediating process was based primarily on the design team's observations of the activity as it occurred. The Virtual Hospital ends with a large group discussion, led by a recognized clinical expert in radiation oncology. The SME chooses each small interdisciplinary team to present their answer to one of the case study questions to the entire group and provides feedback on their responses. During all three years, the design team watched all group discussions and noted no major issues with the treatment decisions that teams made; this was confirmed by conversations with the Virtual Hospital SME and other SMEs present for the discussion such as keynote speakers. A related finding also emerged from the qualitative survey data; participants were asked what they liked most about the $\mathrm{VH}$, and $19 \%$ identified the case-based format (tied with exposure to other perspectives at $19 \%$ and second to active participation at $33 \%$ ). While this doesn't directly support address the conjecture, it does suggest that many participants valued the opportunity to engage in evidence-based treatment planning. Because this aspect was deemed as successful in Year 1 and continued to be supported in Years 2 and 3, no alterations were necessary to the $\mathrm{VH}$ in relation to evidencebased decision-making from the initial Year 1 design.

The second conjecture concerned the participation of all disciplines in team interactions during the $\mathrm{VH}$. The teams were structured so that every discipline was represented by at least one participant, but most included multiple biologists, physicists, and clinicians. While it was possible for the designers and SMEs to observe the teams as they interacted, it was not possible to directly track participation by discipline in each group throughout the activity in light of resource constraints (including the layout of the room). However, because we knew that every team included multiple representatives from each discipline, we could roughly estimate participation by monitoring the number of people who were engaged in team discussions. While there were certainly isolated instances of particular group members participating less than others, there were no indications that this was discipline-specific. SMEs at the course were more familiar with participants' disciplinary backgrounds and also noted no major concerns with a lack of participation by particular disciplines.

We also turned to qualitative data from the participant survey; including what they liked most about the Virtual Hospital and what suggestions they had to improve it. Among the most liked aspects of the $\mathrm{VH}$, the most common response was active participation at $33 \%$ followed by exposure to other perspectives at $19 \%$ (as mentioned, this was tied with the case-based format). Taken together, then, most participants identified one of the two primary aspects of this conjecture (interdisciplinary participation) as being their favorite part of the activity. Although there was variance in percentages across years, these two aspects were among the most commonly mentioned in all three years. 
Finally, we also looked at participant suggestions for improving the activity. It should be noted that most participants either did not respond to this question or left a general positive comment, so percentages reflect the percentage of suggestions given rather than the percentage of participants. In Years 1, their suggestions focused primarily on content issues such as the data included in the case study or the case topic (31\% of all suggestions), while only $8 \%$ of Year 1 suggestions mentioned any issues with their group at all. In Year 2, the percentage of suggestions relating to group issues rose to $20 \%$, although this category included interpersonal issues as well as notes about uneven participation of the disciplines. Because of this, the design team opted to make no changes to the design for Year 3 in relation to interdisciplinary participation but instead opted to closely monitor this aspect. In Year 3, case study questions were revised based on the strong convictions of a new clinical SME who maintained the case questions needed to be more clinical focused. Unfortunately, the changes resulted in more issues with equitable participation, as $32 \%$ of Year 3 suggestions were related to group issues with several specifying that the case study questions were too focused on the clinician's perspective without integrating the other disciplines. As a consequence, the design team will be incorporating redesigned questions designed with the input and agreement of all three SMEs (physicist, biologist and clinician) in the redesign in the case study for Year 4. However, we do believe that the overall design of the activity resulted in the intended mediating process participation and integration of all disciplines in team collaboration.

\section{Learning Outcomes: Results and Discussion}

Having established that the expected mediating processes did occur, next we will consider the learning outcomes that we believed participants would reach as a result of those mediators. These included building or strengthening interdisciplinary communication skills and understanding the value and contribution of all three fields to radiation oncology treatment. These outcomes are being measured over the long term by looking at evidence such as the number and type of interdisciplinary collaborations that participants engage in after leaving the program. At least one study has demonstrated that interdisciplinary science research teams produce fewer publications initially - but more over time - than traditional single-discipline research, so it will be several years before we can collect enough data from participants to draw a conclusion about the impact of the course let alone its individual activities.

However, for the purposes of the learning design, we can draw on observational data from the large group discussion for a reasonable approximation of these learning outcomes within the context of the activity. Based on our design team and SME notes, the small teams in each year were able to provide responses to the case study questions that were in line with expectations for an interdisciplinary approach to solving the problem; in other words, the answered they provided suggested that every discipline had participated. We also considered the finding mentioned previously, that active participation (included interaction and discussion with colleagues from other disciplines) was the favorite feature of the $\mathrm{VH}$ most commonly mentioned by participants. While this is relying on their perceptions of the activity versus actual evidence of learning, participants must be engaging in interdisciplinary discussions before they can claim to enjoy them so for the purposes of the design we felt it was an important data point. Overall, based on this evidence, the design team made no changes to the VH in relation to the first learning outcome. 
The second learning outcome, understanding the value and contribution of all three fields to radiation treatment, is again best measured via long-term collaborative outputs. However, because the learning outcome is in part based on influence participants' attitudes about the value of interdisciplinary work, we can also draw on participant survey responses to make design decisions regarding this learning outcome. The participant responses regarding the most liked aspects of the activity would again be relevant, as exposure to other perspectives and disciplines was the second most common feature mentioned at $19 \%$. We also considered responses from the question that asked participants to describe the experience of the $\mathrm{VH}$, and over $50 \%$ of the total descriptors referenced either a positive interaction aspect (such as interacting with people in other fields) or a positive cognitive aspect (including exposure to content from multiple disciplines). The design team viewed these results as providing sufficient evidence that participants were making progress on this learning outcome, so no alterations were made to the Virtual Hospital relative to participants' understanding of the value of interdisciplinary work in radiation oncology.

\section{Recommended Design Principles for Interdisciplinary Design Teams}

This complex design problem required the application of numerous design principles to organize and support the functions of the interdisciplinary design team. The following is list of guiding principles that emerged during the design and implementation of the VH. These recommendations are not a generic set of principles directly transferable to other contexts; rather it is a list of principles that may be used to improve practice and guide other interdisciplinary design teams.

1. Include key stakeholders from each identified discipline to work jointly to develop and use a shared conceptual framework to create new approached to address the design problem. For this case we employed the Layers of Negotiation Framework.

2. Ensure each interdisciplinary team member has the commitment to devote substantial time to the design effort. For this case, we met for a minimum of 2 hours weekly for 9 months to design the intervention. Each member was required to meet weekly for this collaborative effort.

3. Employ a participatory, inclusive and effective communication model. Using Layers of Negotiation provided a space for interdisciplinary team discussion, reflection, an iterative examination of the design ideas and a process for consensus building.

4. Create a structure to establish a hospitable space promoting mutual respect among team members. Our design space included an agenda initiated by the design Co-PI then sent to all team members one day before the weekly meeting with room for additional agenda items agreed upon before the meeting. The interdisciplinary collaborations fostered the integration of ideas, theories and models from the different disciplines.

5. Employ team learning. Weekly in person team meetings fostering reflection, action, feedback and discussion improved interdisciplinary team learning resulting in an interdisciplinary designed intervention.

6. Create and maintain an interdisciplinary definition document. Each team member has differences in professional language, traditions and values. The creation and maintaining of our interdisciplinary definition document was an educational tool for each team member fostering an appreciation for and learning in and about other disciplines. This also supported the team in creating a shared language. 
7. Ensure a technological infrastructure to support team activities. The team used Google applications to design, store and communicate, a system all were familiar with and had easy access to.

\section{Recommended Design Principles for Interdisciplinary Educational Interventions}

The following is a list of guiding principles used during the design and implementation of the interdisciplinary learning intervention, the Virtual Hospital. These recommendations may improve practice and help other design teams design productive and meaningful engagements in interdisciplinary instructional interventions.

1. Engage learners in solving real world interdisciplinary problems. The Virtual Hospital activity focused on a real-world problem; a patient in need of interdisciplinary cancer treatment care. Expertise from participants in each discipline was necessary to correctly solve the case.

2. Create interdisciplinary participant teams based on participant existing knowledge. Participant course registration included survey questions providing the design team with a snapshot of each participant's existing knowledge and experience. The design team then created participant teams in part based on this snapshot. While an individual participant may not be able to completely solve the case, the collective knowledge from the group could solve the problem.

3. All aspects of the design and redesign efforts must include the entire interdisciplinary design team to ensure meaningful engagement. As previously stated, year 3 evaluation data indicated participants were uninterested with the Virtual Hospital questions by the end of the week. The design team altered the questions for Year 3 to unique case specific questions rather than generic. The clinical SME revised the questions and the remainder of the team did not fully consider the impact these revisions might have on the physicist and biologist participants. The changes resulted in more issues with equitable participation, with several specifying that the case study questions were too focused on the clinician's perspective without integrating the other disciplines. The redesign of these questions will include the efforts and knowledge of all of the SMEs in order to increase interdisciplinary participant engagement.

4. Activities must include a modeling component to support synthesis. The Virtual Hospital included a large group discussion at the end of the activity led by a SME who provided feedback and guidance to each team based on their responses to specific case questions. Structuring the activity to include this modeling promoted a synthesis of content from each individual discipline.

5. Active learning activities promote interdisciplinary engagement. Designing the Virtual Hospital activity where learners were required to work together in order to solve the case promoted engagement between the individual disciplines while encouraging learning in a realistic and relevant situation.

6. Structure activities to include direct and indirect interaction. The Virtual Hospital activity provided scaffolding through the interactive navigation buttons, decision opportunities while navigating through the case and with the final case questions. These direct and indirect interaction occasions promoted team interaction as members worked together navigating through the case. 


\section{Conclusion}

Collaborative activities such as the Virtual Hospital foster interdisciplinary collaboration with the intent of providing a common language and understanding of each of the three disciplines in radiation oncology. One result of the design and evaluation of this collaborative educational activity is a list of recommended design principles for interdisciplinary design teams and for the design of interdisciplinary educational interventions. We hope other interdisciplinary design teams find these recommended design principles useful as they embark on interdisciplinary design.

\section{Acknowledgement}

Research reported in this publication was supported by the Cancer Education Grants Program of the National Cancer Institute at the National Institutes of Health under award number R25CA171971. The content is solely the responsibility of the authors and does not necessarily represent the official views of the National Institutes of Health.

\section{References}

Abercrombie, M. L. J. (1960. The anatomy of judgment. Developmental Medicine and Child Neurology, 2(1), 45-49.

Brereton, M. F., Cannon, D. M., Mabogunje, A. \& Leifer, L .J. (1996). Collaboration in design teams: How social interaction shapes the product. In N. Cross, H. Christiaans, \& K. Dorst (Eds.) Analysing design activity (pp.319-341). Chichester: John Wiley and Sons.

Brown, T. (2009). Change by design. New York: Harper Collins.

Buffee, K. A. (1984). Collaborative learning and the "conversation of mankind". College English, 46(7), 635-652.

Cennamo, K. (2003). Design as knowledge construction: Constructing knowledge of design. Computers in the Schools, 20(4), 13-35.

Cross, D. (2011). Design thinking. New York: Berg.

Dynlacht, J. R., Zeman, E. M., Held, K. D., Deye, J., Vikram, B., \& Joiner, M. (2015). Education and training needs in the radiation sciences: Problems and potential solutions. Radiation Research, 184, 449-455.

Hirsch, A. E., Bishop, P. M., Dad, L., Singh, D., \& Slanetz, P. J. (2009). An increase in medical student knowledge of radiation oncology: A pre-post examination analysis of the oncology education initiative. International Journal of Radiation Oncology Biology Physics, 73(4), 1003-1008.

Januszewski, A. \& Molenda, M. (2008). Education technology: A definition with commentary. New York: Routledge.

Karagiorgi, Y. \& Symeou, L. (2005). Translating constructivism into instructional design: Potential and limitations. Educational Technology \& Society, 8(91), 17 -27.

Lichtman, M. (2006). Qualitative research in education: A user's guide. Thousand Oaks, CA: Sage. 
Nelson, H.G. \& Stolterman, E. (2012). The design way. Cambridge, MA: The MIT Press.

Perkins, D. N. (1992). Technology meets constructivism: Do they make a marriage? In T. M. Duffy \& D. H. Jonassen (Eds.), Constructivism and the technology of instruction: A conversation (pp.45-55). Mahwah, NJ: Lawrence Erlbaum Associates.

Richey, R., Klein, J., \& Tracey, M.W. (2011). The instructional design knowledge base:

Theory, research and practice. New York: Lawrence Erlbaum.

Sandoval, W. (2014). Conjecture mapping: An approach to systematic educational design research. Journal of the Learning Sciences, 23(1), 18-36, DOI: 10.1080/10508406.2013.77824

Smith, B. D., Haffty B. G., Wilson L. D., Smith G. L., Patel A. N., Buchholz T. A. (2010). The future of radiation oncology in the United States from 2010 to 2020: Will supply keep pace with demand? Journal of Clinical Oncology, 28(5) 160-165.

Statwicz, T. \& Stevens, R. (2008). A distributed perspective on collaborative activity. In M. Spector, M. D. Merrill, J. van Merrienboer, \& M. Driscoll (Eds.), Handbook of research on educational communications and technology (3rd ed., pp. 163-171). Mahwah, NJ: Lawrence Erlbaum.

Sullivan, D. C. (2000). Biomedical imaging symposium: Visualizing the future of biology and medicine 1. Radiology, 215(3), 634-638.

Tracey, M. W. (2015). Design team collaboration with a complex design problem. In B. Hokanson, G. Clinton, \& M. Tracey (Eds.) The design of learning experience: Creating the future of educational technology (pp.93-108). New York: Springer.

Zerhouni, E. (2003). NIH Roadmap. Science, 302(5644), 398-399.

Correspondence: Monica W. Tracey, Associate Professor, Learning Design and Technology, College of Education, Wayne State University, Detroit, Michigan, United States 